\title{
Caracterización clínica y microbiológica de episodios de bacteriemia por Streptococcus grupo viridans en niños con cáncer y neutropenia febril de alto riesgo
}

\author{
Clinical characteristics and microbiological profile of viridans group streptococci \\ bacteremia in children with cancer and high-risk febrile neutropenia
}

\author{
Daniel Cortés', María Eliana Maldonado', María Carolina Rivacoba², Verónica de la Maza, Romina Valenzuela³, Ernesto Payá2, \\ Verónica Contardo4, Ana María Álvarez, Carmen Luz Avilés ${ }^{6}$, Ana Becker7, Carmen Salgado², Juan Tordecilla", Mónica Varas 5 , \\ Marcela Venegas ${ }^{6}$, Milena Villarroel $^{3}$, Tamara Viviani ${ }^{7}$, Marcela Zubieta ${ }^{2}$ y María Elena Santolaya ${ }^{3}$
}

\author{
1Programa de título de especialistas en Infectología pediátrica, Facultad de Medicina, Universidad de Chile. \\ ${ }^{2}$ Departamento de Pediatría, Hospital Dr. Exequiel González Cortés, Facultad de Medicina, Universidad de Chile. \\ ${ }^{3}$ Departamento de Pediatría, Hospital Dr. Luis Calvo Mackenna, Facultad de Medicina, Universidad de Chile. \\ ${ }^{4}$ Departamento de Pediatría, Hospital Dr. Roberto del Río, Facultad de Medicina, Universidad de Chile. \\ ${ }^{5}$ Departamento de Pediatría, Hospital San Juan de Dios. \\ ${ }^{6}$ Departamento de Pediatría, Hospital San Borja-Arriarán. \\ ${ }^{7}$ Departamento de Pediatría, Hospital Dr. Sótero del Río.
}

Financiamiento: Proyecto FONDECYT 1161662

Conflictos de interés: Los autores declaran no tener conflictos de interés.

Recibido (segunda versión): 30 de mayo de 2020 / Aceptado: 23 de junio de 2020

\section{Resumen}

Introducción: Streptococcus grupo viridans (SGV) ha adquirido relevancia como microorganismo causante de neutropenia febril, asociándose a morbilidad significativa. Objetivo: Caracterizar episodios de bacteriemia causados por SGV en niños con cáncer que desarrollaron neutropenia febril de alto riesgo (NFAR) desde abril de 2004 a junio de 2018 en seis hospitales pediátricos de Santiago, Chile. Pacientes y Métodos: Análisis retrospectivo de bases de datos de cuatro proyectos FONDECYT sucesivos, prospectivos y multicéntricos, registrando características clínicas y de laboratorio de los pacientes, además de patrón de resistencia antimicrobiana de las cepas aisladas. Resultados: Se registraron 95 episodios de bacteriemia asociada a SGV en 91 niños con NFAR. Destacan: leucemia mieloide aguda como enfermedad de base, neutropenia profunda, hospitalización prolongada (15 días), uso extendido de antimicrobianos (14 días), uso de citarabina en esquemas de quimioterapia ( $86 \%$ episodios). Las manifestaciones clínicas más frecuentes fueron respiratoria y gastrointestinal, asociándose en $26 \%$ a síndrome de shock por Streptococcus grupo viridans. Hubo elevada resistencia a $\beta$ lactámicos, sin cepas no susceptibles a vancomicina. Discusión: SGV es un patógeno relevante en niños con cáncer, fiebre y neutropenia en nuestro medio, asociado a casos de sepsis. La resistencia a $\beta$ lactámicos es un aspecto que requiere vigilancia epidemiológica estricta en esta población.

Palabras clave: Streptococcus grupo viridans; neutropenia febril; bacteriemia. 


\section{Introducción}

S treptococcus grupo viridans (SGV) es un grupo heterogéneo de microorganismos grampositivos, pertenecientes al género Streptococcus, constituyentes del microbioma normal humano, localizados de manera predominante en la cavidad oral y en el tracto respiratorio superior, tracto gastrointestinal y mucosa genital ${ }^{1}$. Este grupo de bacterias ha adquirido relevancia como microorganismos patógenos en población inmunocomprometida, principalmente en pacientes con trasplante de precursores hematopoyéticos y neoplasias hematológicas 2 . Las especies de relevancia dentro del grupo SVG son Streptococcus grupo mitis, con nueve especies, Streptococcus grupo mutans, con ocho especies, Streptococcus grupo anginosus, con tres especies, Streptococcus grupo bovis, con cuatro especies y Streptococcus grupo salivarius, con tres especies ${ }^{3}$.

Diversos investigadores han reportado un incremento de infecciones por microorganismos grampositivos en pacientes con cáncer y neutropenia febril (NF) en tratamiento quimioterápico, siendo relevante dentro de éstos la presencia de $\mathrm{SGV}^{4}$. Se han descrito como factores de riesgo para el aumento de la incidencia de SGV el empleo de citarabina en altas dosis en el esquema de quimioterapia, la mucositis intensa, períodos de neutropenia prolongada y tener como enfermedad de base leucemia mieloide aguda ${ }^{5}$. Las infecciones por estos agentes han requerido atención en esta población por la gravedad del cuadro clínico, que en ocasiones se expresa como síndrome de shock asociado a SGV, determinando hospitalizaciones en Unidades de Paciente Crítico y letalidad ${ }^{6}$. Actualmente, existen reportes de aumento de la resistencia a antimicrobianos en las especies incluídas dentro de SGV, particularmente a $\beta$-lactámicos como penicilinas y cefalosporinas, generando debate respecto a la decisión de terapia empírica que incluya glicopéptidos en este tipo de infecciones?

El objetivo de este estudio fue describir las características clínicas y microbiológicas de episodios de $\mathrm{NF}$ en niños con cáncer, con bacteriemia causada por SGV.

\section{Pacientes y Métodos}

\section{Diseño global de estudio}

Estudio retrospectivo, en que se analizaron los episodios de bacteriemia por SGV de pacientes bajo 18 años de edad, con cáncer y episodios de NF de alto riesgo (NFAR) posteriores a sus ciclos de quimioterapia, provenientes de las bases de datos obtenidas de cuatro proyectos FONDECYT sucesivos, prospectivos y multicéntricos, realizados entre los años 2004 y 2018 (FONDECYT 1040907, 1090194, 1120800 y 1161662) en seis hospitales públicos de Santiago de Chile, pertenecientes a la red del Programa Infantil Nacional de Drogas Antineoplásicas (PINDA).
Los centros participantes en este estudio corresponden a los hospitales: Dr. Luis Calvo Mackenna, Dr. Roberto del Río, Dr. Exequiel González Cortés, San Juan de Dios, Dr. Sótero del Río y San Borja-Arriarán.

Cada uno de los respectivos proyectos contó con las necesarias aprobaciones de los Comité de Ética y de los directores de los hospitales participantes, ingresando a cada estudio los pacientes previo consentimiento informado de los padres o tutores legales y asentimiento de los niños mayores de 11 años.

Para la elección de los casos, se revisaron las bases de datos de los proyectos descritos y se seleccionaron aquellos episodios con aislamiento de SGV en hemocultivos (HC) centrales y periféricos, recolectándose variables demográficas, clínicas y de laboratorio: edad, sexo, tipo de cáncer, uso de profilaxis antimicrobiana, régimen de quimioterapia previo al episodio, número de días entre la última quimioterapia y el inicio de la fiebre; estado general del paciente, presencia de foco clínico, compromiso de mucosas, presencia de sepsis; recuento mínimo de neutrófilos y valor máximo de proteína $\mathrm{C}$ reactiva $(\mathrm{PCR})$ durante la evolución del episodio.

En cada episodio de NFAR se obtuvieron HC periféri$\cos$ (venopunción) y de catéter venoso central (CVC), los que fueron analizados por sistema automatizado en cada centro participante, utilizando sistema BacT/ALERT ${ }^{\circledR}$ (bioMerieux, Inc, Durham, NC, USA). Para la identificación del microorganismo aislado se empleó estudio fenotípico tradicional en el primer período (2004-2008) y sistema VITEK2 ${ }^{\circledR}$ (bioMerieux) desde 2009 en adelante. El estudio de resistencia a antimicrobianos fue determinado por epsilometría E-test ${ }^{\circledR}$ (bioMerieux), empleándose puntos de corte establecidos por CLSI M100. La anotación de variables demográficas, clínicas y de laboratorio se realizó de manera descriptiva para la muestra completa, con información recolectada en forma protocolizada en cada uno de los proyectos incluidos en este estudio. El análisis de resistencia a antimicrobianos se realizó considerando los cuatro períodos incluidos en el estudio (2004-2008; 2009-2012; 2013-2015 y 2016-2018), comparando el patrón de resistencia a penicilina y a cefalosporinas de tercera generación y la no susceptibilidad a vancomicina en cada período de tiempo.

\section{Definiciones}

Neutropenia: recuento de neutrófilos $\leq 500$ céls $/ \mathrm{mm}^{3}$ $\mathrm{o}<\mathrm{a} 1.000 / \mathrm{mm}^{3}$ si se espera una disminución a $<500 /$ $\mathrm{mm}^{3}$ en las siguientes $24-48 \mathrm{~h}$.

Fiebre: temperatura axilar $\geq 38^{\circ} \mathrm{C}$ en dos mediciones separadas por $1 \mathrm{~h}$ o una medición $\geq 38,5^{\circ} \mathrm{C}$.

NFAR: episodio de NF que cumple uno de los siguientes criterios: diagnóstico de leucemia no linfoblástica, presencia de hipotensión arterial, $\mathrm{PCR} \geq 90 \mathrm{mg} / \mathrm{L}, \mathrm{o}$ los siguientes dos criterios: número de días desde el último 


\section{Características clínicas y de laboratorio}

En 40 episodios $(42 \%)$ se documentó bacteriemia sin foco, en 29 episodios (31\%) el foco clínico fue respiratorio, desde infección respiratoria alta hasta neumonía, de los cuales 30\% (n: 9) evolucionó a SDRA y requirió ventilación mecánica. En 19 episodios (20\%) hubo manifestaciones gastrointestinales, con 15 casos de diarrea aguda y cuatro episodios de tiflitis. Se realizó diagnóstico de síndrome de shock asociado a SGV en 25 episodios (26\%), 20 de los cuales tenían antecedente de uso de citarabina.

Todos los pacientes tuvieron RAN $<100$ céls $/ \mathrm{mm}^{3}$ al ingreso al hospital, destacando parámetros inflamatorios elevados durante el curso de la infección, con una mediana de PCR de $225 \mathrm{mg} / \mathrm{L}$. La mediana de duración de la fiebre fue de seis días, la de hospitalización de 15 días y la de duración de terapia antimicrobiana de 14 días. En 87\% de los episodios se empleó vancomicina, principalmente por persistencia de fiebre pese al uso de $\beta$ lactámicos (Tabla 2).

En esta serie se registraron cuatro muertes, de las cuales una se consideró relacionada a la infección por SGV, en un paciente que evolucionó con shock refractario y falla orgánica múltiple. De los restantes tres casos, en dos la muerte se atribuyó a la enfermedad de base y en un caso a una candidiasis invasora.

\section{Características microbiológicas y resistencia a antimicrobianos}

El informe microbiológico fue de SGV en $100 \%$ de los casos entre los años 2004 y 2008. Del 2009 al 2015,

crobianos y sus respectivos intervalos de confianza utilizó método de Wald modificado, realizando los análisis comparativos de resistencia a penicilina, cefalosporinas de tercera generación y vancomicina entre los períodos incluidos en el estudio con prueba exacta de Fisher. Se utilizó el programa QuickCalcs ${ }^{\circledR}$ de $\operatorname{GraphPad}^{\circledR} 2018$. Se consideró significativo un valor de $\mathrm{p}<0,05$.

\section{Resultados}

\section{Características generales de la población}

Durante el período de estudio, comprendido entre abril de 2004 y junio de 2018, de un total de 2.296 episodios de NFAR, hubo aislamiento microbiológico en 654 de ellos. Se registró un total de 91 niños con 95 episodios de bacteriemia por SGV (14,5\% del total de episodios de bacteriemia).

Las características generales de la población en estudio son descritas en la Tabla 1. La mediana de edad fue de 6 años (pc 25-75: 3 a 10 años), 51\% fueron hombres y $78 \%$ tuvo un cáncer hematológico. Se objetivó empleo de $\beta$ lactámicos profiláctico (amoxicilina) en 13\% de los pacientes y de citarabina como fármaco de quimioterapia previo al episodio de NFAR en 82 episodios (86\%).

\begin{tabular}{|c|c|}
\hline \multicolumn{2}{|l|}{ Características de los pacientes $(n=91)$} \\
\hline Edad (años). Mediana (p25-75) & $6(3-10)$ \\
\hline Sexo masculino (\%) & $46(51)$ \\
\hline \multicolumn{2}{|l|}{ Tipo de cáncer. N (\%) } \\
\hline Leucemia mieloide aguda & $46(50)$ \\
\hline Leucemia linfoblástica aguda & $18(20)$ \\
\hline Recaída de leucemia & 7 (8) \\
\hline Tumor del sistema nervioso central & $10(11)$ \\
\hline Recaída de tumor sólido & $3(3)$ \\
\hline Otros tipos de cáncer & 7 (8) \\
\hline \multicolumn{2}{|l|}{ Características de los episodios $(n=95)$} \\
\hline Número de días desde última QT a inicio fiebre. Mediana (p25-75) & $7(5-8)$ \\
\hline Uso de profilaxis con amoxicilina. N (\%) & $12(13)$ \\
\hline Administración de citarabina en QT. N (\%) & $82(86)$ \\
\hline
\end{tabular}


Tabla 2. Características clínicas y de laboratorio de 95 episodios de bacteriemia por Streptococcus grupo viridans en 91 niños con cáncer, fiebre y neutropenia en 6 hospitales Santiago, Chile, años 2004-2018

\begin{tabular}{|l|l|}
\hline Variable & $\mathbf{n}$ \\
\hline $\begin{array}{l}\text { Foco clínico. N (\%) } \\
\quad \text { Bacteriemia sin foco }\end{array}$ & $40(42)$ \\
$\quad$ Respiratorio alto/bajo & $29(31)$ \\
$\quad \begin{array}{l}\text { Digestivo alto/bajo } \\
\quad \text { Asociado a catéter venoso central } \\
\quad \text { Celulitis }\end{array}$ & $6(20)$ \\
\hline Mucositis. N (\%) & $1 \quad(1)$ \\
\hline RAN (céls/mm) al ingreso. Mediana (pc 25-75) & $43(45)$ \\
\hline Mayor valor de proteína C reactiva (mg/L). Mediana (p25-75) & 0 \\
\hline No de días de hospitalización. Mediana (p25-75) & $225(127-291)$ \\
\hline No de días de fiebre. Mediana (p25-75) & $15(11-19)$ \\
\hline No días de uso de antimicrobianos. Mediana (p25-75) & $6(4-10)$ \\
\hline Uso de vancomicina. N (\%) & $14(11-18)$ \\
\hline Días de uso de vancomicina. Mediana (p25-75) & $83(87)$ \\
\hline Necesidad de soporte ventilatorio. N (\%) & $10(5-13)$ \\
\hline Sepsis. N (\%) & $11(12)$ \\
\hline Ingreso a unidad de paciente crítico. N (\%) & $25(26)$ \\
\hline Mortalidad. N (\%) & $25(26)$ \\
\hline
\end{tabular}

$40 \%$ de los aislados identificó la especie, lo que aumentó a $69 \%$ entre los años 2016 a 2018. De los 95 aislados, 58 fueron informados como SGV. De los restantes 37, la identificación fue: S. mitis/oralis (n: 18), S. mitis (n: 14), S. mutans (n: 2), S. salivarium (n: 2) y S. peroris (n: 1). No hubo diferencia significativa en la presentación clínica ni en el perfil de resistencia entre las distintas especies (P: NS).
Se obtuvo el dato de resistencia antimicrobiana en $84 / 95$ aislados microbiológicos (88\%). Se determinó la resistencia a penicilina, cefalosporinas de tercera generación y la no susceptibilidad a vancomicina considerando los cuatro períodos incluidos en el estudio, sin observar cambios significativos alguno a lo largo del tiempo (Tabla 3), con un promedio de resistencia a penicilina de 58\% (IC 95\% 33-86) y de resistencia a cefalosporinas de tercera generación de $35 \%$ (IC 95\% 7-76). No hubo detección de cepas no susceptibles a vancomicina en nuestra serie.

\section{Discusión}

De manera similar a lo reportado por otros grupos, las bacteriemias por SGV se han constituido en una importante causa de infección en episodios de NF en los últimos años en el medio nacional. En datos publicados por nuestro grupo, SGV se ha situado como la tercera causa en frecuencia de bacteriemia en niños con NFAR después de enterobacterias y Staphylococcus coagulasa negativa, con aislamiento entre $13 \%$ y $17 \%$ del total de bacteriemias con agente identificado, lo que es acorde a cifras internacionales que describen frecuencia de $6 \%$ a $35 \%{ }^{8-10}$

Desde los primeros reportes de series de casos que emergieron por parte del National Cancer Center en Bethesda y en niños provenientes desde el M. D Anderson Cancer Center en Houston ${ }^{11,12}$, comenzó a constituirse un perfil de enfermedad en esta población de riesgo, teniendo como punto en común tener una neoplasia hematológica, principalmente leucemia mieloide aguda, recaída de leucemia, antecedente de trasplante de precursores hematopoyéticos, esquemas intensificados de quimioterapia, especialmente asociados a empleo de altas dosis de citarabina ${ }^{13}$, (presente en $86 \%$ de los casos en esta serie), intensa inmunosupresión, con RAN $\leq 100$ céls $/ \mathrm{mm}^{3}$ ( 0 céls $/ \mathrm{mm}^{3}$ en nuestra experiencia) y diverso grado de disrupción de mucosas (descrito en $45 \%$ de los casos en nuestro estudio).

\begin{tabular}{|c|c|c|c|c|c|c|c|c|c|}
\hline Antimicrobiano & \multicolumn{2}{|c|}{$\begin{array}{c}2004-2008 \\
\text { n: } 21 \\
\% \text { R (IC } 95 \%)\end{array}$} & \multicolumn{2}{|c|}{$\begin{array}{c}2009-2012 \\
\text { n: } 14 \\
\% \text { R (IC95\%) }\end{array}$} & \multicolumn{2}{|c|}{$\begin{array}{c}2013-2015 \\
\text { n: } 28 \\
\% \text { R (IC } 95 \%)\end{array}$} & \multicolumn{2}{|c|}{$\begin{array}{c}\text { 2016-junio } 2018 \\
\text { n: } 21 \\
\% \text { R (IC } 95 \%)\end{array}$} & \multirow{2}{*}{$\begin{array}{l}\mathbf{p} \\
\text { NS }\end{array}$} \\
\hline Penicilina & 71 & $(50-86)$ & 43 & $(33-79)$ & 60 & $(42-76)$ & 57 & $(40-73)$ & \\
\hline Cefalosporinas $3^{\mathrm{a}}$ gen. & 57 & $(37-76)$ & 36 & $(16-61)$ & 18 & $(7-36)$ & 29 & $(14-50)$ & NS \\
\hline
\end{tabular}


detectando como variables significativas el empleo de algún $\beta$ lactámico como profilaxis o tratamiento en los 30 días precedentes o el inicio de bacteriemia por SGV mientras el paciente estaba hospitalizado. Este modelo fue desarrollado en población adulta y no se ha intentado validar en pediatría de manera prospectiva ${ }^{19}$. El elevado empleo de vancomicina en nuestros pacientes, explicado esencialmente por persistencia de fiebre o concomitancia de sepsis más que por el patrón de resistencia, mantiene vigente el debate del uso racional de este antimicrobiano en niños con cáncer y episodios de NFAR. Existen recomendaciones formales sobre el uso de vancomicina en pacientes con NF. La primera es que no se justifica el uso de vancomicina de rutina como terapia empirica inicial en episodios de NFAR, salvo en pacientes que ingresen con sepsis o con sospecha de microorganismos resistentes. La segunda es que no se justifica escalar en la terapia antimicrobiana desde penicilina o cefalosporinas de tercera generación a vancomicina sólo por persistencia de la fiebre en pacientes clínicamente estables ${ }^{20}$.

Finalmente, las bacteriemias por SGV en nuestro medio tienen comportamiento similar a lo descrito en la literatura científica, con factores de riesgo que se replican, desarrollo de morbilidad relevante como síndrome de shock por SGV y patrón de resistencia a $\beta$ láctamicos considerable. Es necesario continuar con una vigilancia epidemiológica activa que contribuya a racionalizar el manejo de este tipo de pacientes.

\section{Referencias bibliográficas}

1.- Tunkel A, Sepkowitz K. Infections caused by viridans streptococci in patients with neutropenia. Clin Infect Dis 2002; 34: 1524-9. doi: 10.1086/340402.

2.- Gamis A S, Howells W B, DeSwarte-Wallace J, Feusner J H, Buckley J D, Woods WG. $\alpha$ hemolytic streptococcal infections during intensive treatment for acute myeloid leukemia: a report from the Children's Cancer Group Study CCG-2891. J Clin Oncol. 2000; 18: 1845-55. doi: 10.1200/JCO.2000.18.9.1845.

3.- Spellerberg B, Brandt C. Chapter 29: Streptococcus. Murray P, Baron E, Jorgensen J, Landry M, Pfaller M, eds. Manual of Clinical Microbiology, $9^{\text {th }}$ ed. Washington DC: ASM Press; 2007, 412-6.

4.- Gudiol C, Bodro M, Simonetti A, Tubau F, González-Barca E, Cisnal M, et al. Changing aetiology, clinical features, antimicrobial resistance and outcomes of bloodstream infection in neutropenic cancer patients. Clin Microbiol Infect 2013; 19: 474-9. https://doi. org/10.1111/j.1469-0691.2012.03879.x.
5.- $\quad$ Paganini H, Staffolani V, Zubizarreta P, Casimir L, Lopardo H, Luppino V. Viridans streptococci bacteremia in children with fever and neutropenia: a case control study of predisposing factors. Eur J Cancer 2003; 39: 1284-9. https:// doi.org/10.1016/S0959-8049(03)00272-7.

6.- Lewis V, Yanofsky R, Mitchell D, Dix D, Ethier MC Gillmeister B, et al. Predictors and outcomes of viridans group streptococcal infections in pediatric acute myeloid leukemia: from the Canadian Infections in AML Research Group. Pediatr Infect Dis J. 2014; 33: 126-9. doi: 10.1097/INF.0000000000000058.

7.- Han S B, Bae E Y, Lee J W, Lee D G, Chung $\mathrm{N} \mathrm{G}$, Jeong D C, et al. Clinical characteristics and antimicrobial susceptibilities of viridans streptococcal bacteremia during febrile neutropenia in patients with hematologic malignancies: a comparison between adults and children. BMC Infect Dis 2013; 13: 273-83. doi: 10.1186/1471-2334-13-273.

8.- Maldonado M E, Acuña M, Álvarez A M, Avilés C, De la Maza V, Salgado C, et al. Microorganismos aislados de hemocultivos en niños con cáncer y neutropenia febril de alto riesgo en cinco hospitales de Santiago, Chile, período 2012-2015. Rev Chilena Infectol 2018; 35 (2): 140-6. http://dx.doi.org/10.4067/s071610182018000200140

9.- Zinner S H. Changing epidemiology of infections in patients with neutropenia and cancer: emphasis on gram-positive and resistant bacteria. Clin Infect Dis 1999; 29: 490-4. doi: 10.1086/598620.

10.- Ahmed R, Hassall T, Morland B, Gray J. Viridans streptococcus bacteremia in children on chemotherapy for cancer: an underestimated problem. Pediatr Haematol Oncol. 2003; 20 : 439-44. PMID: 14631617.

11.- Pizzo P A, Ladisch S, Witebsky F G. Alphahemolytic streptococci: clinical significance in the cancer patient. Med Pediatr Oncol 1978; 4: 367-70. doi: 10.1002/mpo.2950040414.

12.- Hoecker J L, Pickering L K, Groschel D, Kohl S. Streptococcus salivarius sepsis in children with malignancies. J Pediatr 1978; 92: 337-8. doi: 10.1016/s0022-3476(78)800407.

13.- Elting L S, Bodey G P, Keefe B H. Septicemia and shock syndrome due to 
viridans streptococci; a case-control study of predisposing factors. Clin Infect Dis 1994; 14 : 1201-7. doi: 10.1093/clinids/14.6.1201.

14.- Han S B, Bae E Y, Lee J W, Lee D G, Chung N G, Jeong D C, et al. Clinical characteristics and antibiotic susceptibility of viridans streptococcal bacteremia in children with febrile neutropenia. Infection 2013; 41: 917-24. doi: 10.1007/s15010-0130470-7.

15.- Marron A, Carratalà J, González-Barca E, Fernández-Sevilla A, Alcaide F, Gudiol F. Serious complications of bacteremia caused by viridans streptococci in neutropenic patients with cancer. Clin Infect Dis 2000; 31: 1126-30. doi: $10.1086 / 317460$.

16.- Nielsen M J, Claxton S, Pizer B, Lane S, Cooke R P, Paulus S, et al. Viridans group streptococcal infections in children after chemotherapy or stem cell transplantation: A 10-year review from a tertiary pediatric hospital. Medicine (Baltimore) 2016 Mar; 95(9): e2952. doi: 10.1097/ MD.0000000000002952.

17.- Carratalá J, Alcaide F, Fernández-Sevilla A, Corbella X, Liñares J, Gudiol F. Bacteremia due to viridans streptococci that are highly resistant to penicillin: increase among neutropenic patients with cancer. Clin Infect Dis 1995; 20: 1169-73. doi: 10.1093/ clinids/20.5.1169.

18.- Spanik S, Trupl J, Kunova A, Botek R, Sorkovska D, Grey E, et al. Viridans streptococcal bacteraemia due to penicillinresistant and penicillin-sensitive streptococci: analysis of risk factors and outcome in 60 patients from a single cancer centre before and after penicillin is used for prophylaxis. Scand J Infect Dis 1997; 29: 245-9. doi: 10.3109/00365549709019036.

19.- Shelburne S, Lasky R, Sahasrabhojane P, Tarrand J, Rolston K. Development and validation of a clinical model to predict the presence of $\beta$-lactam resistance in viridans group streptococci causing bacteremia in neutropenic cancer patients. Clin Infect Dis 2014; 59 (2): 223-30. doi: 10.1093/cid/ciu260.

20.- Lehrnbecher T, Robinson P, Fisher B, Alexander S, Ammann R, Beauchemin M, et al. Guideline for the management of fever and neutropenia in children with cancer and hematopoietic stem-cell transplantation recipients: 2017 update. J Clin Oncol 2017; 35 (18): 2082-94. doi: 10.1200/JCO.2016.71.7017. 